05,11

\title{
Фаза Имри-Ма в нанокристаллическом ферромагнетике
}

\author{
() А.А. Берзин ${ }^{1}$, А.И. Морозов ${ }^{2}$, А.С. Сигов ${ }^{1}$ \\ ${ }^{1}$ Московский технологический университет (МИРЭА), \\ Москва, Россия \\ ${ }^{2}$ Московский фризико-технический институт (государственный университет), \\ Долгопрудный, Россия \\ E-mail: mor-alexandr@yandex.ru
}

(Поступила в Редакцию 28 февраля 2018 г.

В окончательной редакции 13 марта 2018 г.)

\begin{abstract}
Изучена возможность возникновения неупорядоченного состояния Имри-Ма, индуцированного флуктуациями направления легкой оси анизотропии в нанокристаллическом образце для случая слабого обменного взаимодействия между кристаллитами. Получена фазовая диаграмма системы в переменных „характерный размер кристаллита-обменный интеграл межкристаллитного взаимодействия““. Сделаны оценки характерной величины статистических флуктуаций кристаллографической анизотропии и найдены зависимости коэрцитивного поля от размера кристаллитов для систем разной пространственной размерности.
\end{abstract}

Работа поддержана Минобрнауки России (госзадание, проект № 8.1183.2017 ПЧ).

DOI: 10.21883/FTT.2018.09.46385.050

\section{1. Введение}

В работе [1] была развита теория случайной анизотропии для аморфных ферромагнетиков, в которых анизотропия типа „легкая ось“ изменяется случайным образом на структурной корреляционной длине $D$.

Статистические флуктуации анизотропии на масштабе $L \gg D$ приводят к невыгодности однородного ферромагнитного состояния в пространстве размерности $d<4$ и возникновению неупорядоченного состояния, в котором направление вектора намагниченности следует за локальным направлением легкой оси. Это происходит в полном соответствии с аргументами Имри и Ма [2], сформулированными для систем, содержащих дефекты типа „случайное локальное поле“. На полную аналогию между этим состоянием и состоянием Имри-Ма указывалось в работе [3].

Герцер [4] применил теорию случайной анизотропии к нанокристаллическим ферромагнетикам, в которых роль структурной корреляционной длины играет характерный размер кристаллита $R$.

Полученная оценка для выигрыша объемной плотности энергии $w$ неоднородного состояния, которое в дальнейшем будем называть фазой Имри-Ма, по сравнению с энергией упорядоченного состояния была использована в $[1,4]$ для оценки коэрцитивного поля согласно формуле

$$
H_{c} \sim \frac{|w|}{M_{s}}
$$

где $M_{s}$ - величина намагниченности насыщения. На основе этой оценки в [4] была получена степенная зависимость коэрцитивного поля от размера кристаллита $H_{c} \propto R^{6}$.

В монографии [5] указывается, что в ряде сплавов наблюдается степенная зависимость коэрцитивного по- ля от размера кристаллита с меньшим показателем: $H_{c} \propto R^{3}$. Для ее объяснения в работах [6,7] была предложена модель, в которой предполагалось наличие как случайной анизотропии, так и однородной по объему образца одноосной анизотропии, намного превосходящей случайную. Величина коэрцитивного поля определяется суммарной энергией анизотропии, поэтому в данном случае слагаемое в $H_{c}$, пропорциональное $R^{3}$, должно быть малой добавкой к постоянной величине. Это означает, что предложенная модель противоречит экспериментальным данным, свидетельствующим об изменении коэрцитивного поля на несколько порядков при изменении размера кристаллитов $[8,9]$.

В данной работе рассматривается случайная анизотропия в нанокристаллическом ферромагнетике, у которого обменное взаимодействие между кристаллитами намного слабее, чем в объеме кристаллитов. Это может быть связано как со структурой границ (наличие аморфной фазы), так и с различием химического состава в объеме и в граничных областях кристаллитов. Будут рассмотрены ферромагнетики с высоким фактором качества, для которых кристаллографическая анизотропия типа „легкая ось“ намного превосходит анизотропию формы, которой в дальнейшем будем пренебрегать.

Будет показано, что в объемных образцах таких ферромагнетиков коэрцитивное поле пропорционально кубу размера кристаллита: $H_{c} \propto R^{3}$.

\section{2. Энергия системы спинов}

Пусть ферромагнитное обменное взаимодействие ближайших друг к другу единичных классических спинов (их модули могут быть включены в соответствующие константы) описывается обменным интегралом $J_{0}$, а об- 
менное взаимодействие ближайших друг к другу спинов, принадлежащих соседним кристаллитам, - обменным интегралом $\tilde{J}$.

Энергия одноионной анизотропии имеет вид

$$
W_{a n}=-\frac{1}{2} K_{0} \sum_{\alpha} \sum_{i \in V_{\alpha}}\left(\mathbf{s}_{i, \alpha} \mathbf{e}_{\alpha}\right)^{2},
$$

где $K_{0}$ - константа кристаллографической анизотропии в объеме кристаллита, $\mathbf{s}_{i, \alpha}-i$-й спин, принадлежащий кристаллиту под номером $\alpha, V_{\alpha}$ - объем этого кристаллита, $\mathbf{e}_{\alpha}-$ единичный вектор, задающий направление легкой оси в данном кристаллите.

Толщина доменной стенки (блоховской, неелевской или гибридной) и ее поверхностное натяжение по порядку величины равны

$$
\begin{gathered}
\Delta \sim b \sqrt{\frac{J_{0}}{K_{0}}}, \\
\varepsilon \sim b^{-2} \sqrt{J_{0} K_{0}},
\end{gathered}
$$

где $b$ - межатомное расстояние.

Рассмотрим поведение системы на основе предложенной модели.

\section{3. Область коллективного поведения намагниченностей кристаллитов}

В области больших размеров кристаллитов намагниченность в объеме каждого из них параллельна легкой оси данного кристаллита (индивидуальное поведение намагниченностей кристаллитов). При этом дальний порядок на размере $L \gg R$ отсутствует. Если обменное взаимодействие между кристаллитами того же порядка, что и в объеме кристаллита, то на границах между кристаллитами формируются доменные стенки. Следовательно, критическим значением размера кристаллита $R_{c r}$ является толщина доменной стенки $\Delta$. При $R \ll R_{c r}$ доменные стенки на границах между кристаллитами не могут сформироваться, и имеет место коллективное поведение намагниченностей нанокристаллитов.

В случае слабой связи между кристаллитами при $\tilde{J} \ll \sqrt{J_{0} K_{0}}$ энергетически более выгодным является разворот намагниченности на атомном масштабе $b \ll \Delta$ в зазоре между кристаллитами. Критический размер $R_{c r}$ находится из условия равенства энергии анизотропии в объеме кристаллита $K_{0}(R / b)^{3}$ и энергии неоднородности на границе кристаллита $\tilde{J}(R / b)^{2}$

$$
R_{c r} \sim \frac{\tilde{J b}}{K_{0}} \sim \frac{\tilde{J}}{\sqrt{J_{0} K_{0}}} \Delta \ll \Delta .
$$

При большой энергии анизотропии, когда $K_{0} R \gg \tilde{J} b$, имеет место индивидуальное, а в противоположном случае — коллективное поведение намагниченностей кристаллитов.

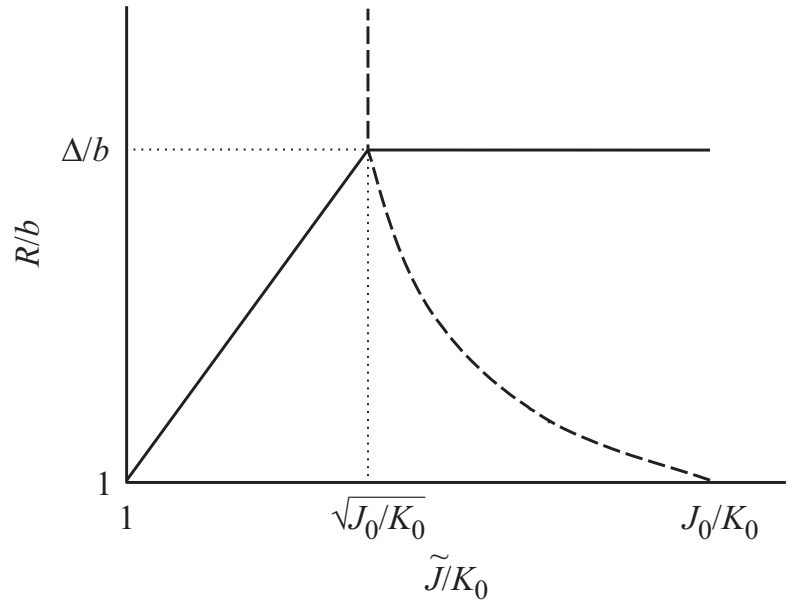

Фазовая диаграмма нанокристаллической системы в переменных „характерный размер кристаллита-обменный интеграл межкристаллитного взаимодействия“". Сплошная линия граница между областями индивидуального (выше линии) и коллективного поведения кристаллитов. Пунктирная линия разделяет области сильного (правее линии) и слабого взаимодействия кристаллитов.

Фазовая диаграмма системы в переменных „характерный размер кристаллита $R$-обменный интеграл межкристаллитного взаимодействия“ приведена на рисунке. Граница между индивидуальным и коллективным поведением намагниченностей кристаллитов изображена сплошной линией. Выше нее наблюдается индивидуальное, а ниже - коллективное поведение. Переход от одного типа поведения к другому происходит непрерывно по мере изменения параметров системы.

\section{4. Энергия неоднородности и механизмы разворота намагниченности}

Характерная величина константы кристаллографической анизотропии типа „легкая ось“, возникающей за счет статистических флуктуаций в области с характерным линейным размером $L \gg R$ составляет величину порядка $[1,4]$ :

$$
\langle K\rangle \sim \frac{K_{0}}{\sqrt{N}} \sim K_{0}\left(\frac{R}{L}\right)^{d / 2},
$$

где $N \sim(L / R)^{d}-$ число кристаллитов в указанной области, а $d-$ размерность пространства. Если намагниченность следует за флуктуациями легкой оси анизотропии, то возникает отрицательный вклад в объемную плотность энергии. По порядку величины этот вклад составляет

$$
w_{a n} \sim-\frac{K_{0}}{b^{d}}\left(\frac{R}{L}\right)^{d / 2} .
$$

Оценим положительный вклад в объемную плотность энергии, возникающий при таком поведении намагничен- 
ности вследствие неоднородности параметра порядка. Если разворот намагниченности происходит непрерывно на масштабе $L$ (первый механизм разворота), то по порядку величины

$$
w_{e x} \sim \frac{J_{0}}{b^{d-2} L^{2}} .
$$

Действительно, градиент угла разворота вектора намагниченности $|\nabla \theta| \sim L^{-1}$, так как на длине $L$ происходит разворот на угол порядка радиана, а $w_{e x} \sim J_{0}(\nabla \theta)^{2} b^{2-d}$. Легко видеть, что при $d<4$ выигрыш в энергии анизотропии превосходит в области больших значений $L$ проигрыш в обменной энергии, обусловленный неоднородностью параметра порядка, и фаза Имри-Ма является основным состоянием системы классических спинов.

Разворот намагниченности может происходить „ступенчато“ путем последовательных скачков на границах кристаллитов (второй механизм разворота). Число таких ступеней на длине $L$ равно $L / R$, а угол разворота на каждой границе кристаллитов порядка $R / L$. Поскольку ширина области контакта кристаллитов атомного масштаба, то $|\nabla \theta| \sim R / L b$, а объемная плотность энергии неоднородности в области контакта

$$
w_{e x}^{(1)} \sim \frac{\tilde{J} R^{2}}{b^{d} L^{2}} .
$$

Относительная доля контактных областей порядка $b / R$. В итоге средняя объемная плотность энергии неоднородности

$$
w_{e x} \sim \frac{\tilde{J R}}{b^{d-1} L^{2}} .
$$

Сравнивая выражения (8) и (10), находим, что первый механизм разворота является преобладающим при $\tilde{J R} \gg J_{0} b$, а второй - при обратном соотношении.

Граница между областями, в которых преобладает один из механизмов разворота, изображена на рисунке пунктирной гиперболой $\frac{R}{b}=\left(\frac{J_{0}}{K_{0}}\right)\left(\frac{\tilde{J}}{K_{0}}\right)^{-1}$. Она является продолжением прямой $\frac{\tilde{J}}{K_{0}}=\sqrt{\frac{J_{0}}{K_{0}}}$, разграничивающей два механизма разворота намагниченности на границах крупных кристаллитов. Переход от одного механизма к другому происходит непрерывным образом по мере изменения параметров.

a) Первый механизм разворота. Минимизируя по $L$ сумму энергий (7) и (9), находим оптимальный масштаб $L^{*}$ и соответствующий выигрыш в объемной плотности энергии по сравнению с однородно намагниченным состоянием, впервые полученные для произвольной размерности пространства в работе [10]:

$$
\begin{gathered}
L^{*} \sim \Delta\left(\frac{\Delta}{R}\right)^{\frac{d}{4-d}}, \\
w^{*} \sim-\frac{K_{0}}{b^{d}}\left(\frac{R}{\Delta}\right)^{\frac{2 d}{4-d}} .
\end{gathered}
$$

б) Второй механизм разворота. Аналогично, минимизируя по $L$ сумму энергий $(7)$ и $(10)$, находим

$$
\begin{gathered}
\tilde{L}^{*} \sim\left(\frac{\tilde{J} b}{K_{0}}\right)^{\frac{2}{4-d}} R^{\frac{2-d}{4-d}}, \\
\tilde{w}^{*} \sim-\frac{K_{0}}{b^{d}}\left(\frac{R K_{0}}{b \tilde{J}}\right)^{\frac{d}{4-d}} .
\end{gathered}
$$

Существенное отличие зависимостей, полученных для первого и второго механизмов разворота, обусловливает качественно разное поведение системы в областях слабого и сильного взаимодействия кристаллитов.

\section{5. Коэрцитивное поле}

Легко видеть, что в области слабого взаимодействия кристаллитов зависимость коэрцитивного поля от размера кристаллита существенно отличается от таковой в области их сильного взаимодействия. Вместо зависимостей $H_{c} \propto R^{2 / 3}, H_{c} \propto R^{2}, H_{c} \propto R^{6}$, полученных в [10] в приближении сильного взаимодействия кристаллитов для одномерных, двухмерных и трехмерных структур, в области слабого взаимодействия кристаллитов получаются соответственно зависимости $H_{c} \propto R^{1 / 3}, H_{c} \propto R$, $H_{c} \propto R^{3}$. Таким образом, наблюдаемая в эксперименте $[5,8,9]$ зависимость $H_{c} \propto R^{3}$ объясняется слабой связью между кристаллитами. При этом в рассматриваемой модели, в отличие от работ [6,7], отсутствует однородная по объему образца сильная одноосная анизотропия. Это позволяет получить существенное изменение коэрцитивного поля с изменением размера кристаллита.

Зависимость $H_{c} \propto \tilde{w}^{*} \propto \tilde{J}^{-\frac{d}{4-d}}$ объясняет рост коэрцитивного поля с увеличением температуры, обнаруженный в работе [11]. Действительно, величина $\tilde{J}$ падает по мере приближения к температуре Кюри аморфной фазы, расположенной в зазоре между кристаллитами. Рост $H_{c}$ продолжается вплоть до нарушения условия $K_{0} R \ll \tilde{J} b$ и перехода в фазу индивидуального поведения намагниченностей кристаллитов.

\section{6. Степень текстурированности}

Приведенная на рисунке фазовая диаграмма справедлива в отсутствие текстурированности образцов, то есть в отсутствие анизотропии в распределении направлений легких осей в пространстве параметра порядка. Как показано в работах [12,13], наличие преимущественного направления и возникновение глобальной (средней) анизотропии типа „легкая ось“ приводит к подавлению состояния Имри-Ма и восстановлению дальнего порядка в случае, когда она превосходит критическое значение [14].

Аналогично [12] можно оценить критическую степень $\varepsilon$ асимметрии распределения $\rho\left(\mathbf{e}_{\alpha}\right)$ направлений легких осей в пространстве параметра порядка, при 
превышении которой состояние Имри-Ма становится энергетически невыгодным. Пусть $\rho\left(\mathbf{e}_{\alpha}\right)$ задается выражением

$$
\rho\left(\mathbf{e}_{\alpha}\right)=A\left[(1+\varepsilon) e_{\alpha 1}^{2}+\sum_{i=2}^{n} e_{\alpha i}^{2}\right],
$$

где $n$ - число компонент параметра порядка, а $e_{\alpha i}-$ проекция вектора $\mathbf{e}_{\alpha}$ на $i$-ю ось в пространстве параметра порядка. Наличие асимметрии с $\varepsilon>0$ понижает энергию состояния с однородной намагниченностью, параллельной оси 1.

Критическое значение $\varepsilon$ находится из равенства энергии такого состояния и состояния Имри-Ма. В результате получаем

$$
\begin{gathered}
\varepsilon_{c r} \sim\left(\frac{R}{\Delta}\right)^{\frac{2 d}{4-d}}, \quad \tilde{J} R \gg J_{0} b, \\
\varepsilon_{c r} \sim\left(\frac{R K_{0}}{b \tilde{J}}\right)^{\frac{d}{4-d}}, \quad \tilde{J} R \ll J_{0} b .
\end{gathered}
$$

Следует отметить, что точно такое же критическое значение имеет отношение объемной плотности энергии анизотропии формы к величине $K_{0} / b^{d}$.

При $R=0.1 \Delta$ из (16) получаем $\varepsilon_{c r}=10^{-6}$ для $d=3$ и $\varepsilon_{c r}=10^{-2}$ для $d=2$. Для $R K_{0}=0.1 b \tilde{J}$ находим из (17): $\varepsilon_{c r}=10^{-3}$ для $d=3$ и $\varepsilon_{c r}=10^{-1}$ для $d=2$. Легко видеть, что наименее жесткие требования к степени текстурированности имеют место в двумерных образцах со слабым взаимодействием кристаллитов.

В случае тонкой ферромагнитной пленки на немагнитной подложке последняя создает поверхностную анизотропию, а малая величина аспектного отношения собственно пленки - заметную анизотропию формы. Поэтому состояние Имри-Ма следует изучать на ферромагнитных пленках, текстурированных так, чтобы кристаллографические легкие оси кристаллитов были изотропно распределены в плоскости пленки. Подложку следует выбирать аморфной, чтобы она не создавала анизотропии для легкой оси кристаллитов в плоскости пленки.

\section{7. Заключение}

Таким образом, теория случайной анизотропии, развитая ранее в [4] для случая сильного взаимодействия кристаллитов, распространена на случай их слабого взаимодействия.

Для фазовой диаграммы „характерный размер кристаллита-обменный интеграл межкристаллитного взаимодействия“ найдены границы между индивидуальным и коллективным поведением намагниченностей кристаллитов и между режимами их сильного и слабого взаимодействия.

Рассчитаны зависимости коэрцитивного поля от размера кристаллитов в области их слабого взаимодействия для систем разной пространственной размерности.
В случае трехмерной системы теоретическая зависимость совпадает с экспериментальными зависимостями, полученными для ряда нанокристаллических ферромагнитных сплавов.

Получено ограничение на степень текстурированности образцов, при которой можно наблюдать состояние Имри-Ма. Показано, что наиболее перспективными для экспериментального изучения являются квазидвумерные образцы.

Авторы благодарны О.Г. Удалову, обратившему их внимание на возможность реализации состояния ИмриМа в нанокристаллах.

\section{Список литературы}

[1] R. Alben, J.J. Becker, M.C. Chi. J. Appl. Phys. 49, 1853 (1978).

[2] Y. Imry, S.-K. Ma. Phys. Rev. Lett. 35, 1399 (1975).

[3] Р.С. Исхаков, В.А. Игнатченко, С.В. Комогорцев, А.Д. Балаев. Письма в ЖЭТФ 78, 1142 (2003).

[4] G. Herzer. IEEE Trans. Magn. 25, 3327 (1989); 26, 1397 (1990).

[5] А.М. Глезер, Н.А. Шурыгина. Аморфно-нанокристаллические сплавы. Физматлит, М. (2013). 452 с.

[6] K. Suzuki, J.M. Cadogan. Phys. Rev. B 58, 2730 (1998).

[7] K. Suzuki, N. Ito, J.S. Garitaonandia, J.D. Cashion, G. Herzer. J. Non-Cryst. Solids 354, 5089 (2008).

[8] Y. Fujii, H. Fujita, A. Seki, T. Tomida. J. Appl. Phys. 70, 6241 (1991).

[9] D. Yao, S. Ge, X. Zhou, H. Zuo. J. Appl. Phys. 107, 073902 (2010).

[10] G. Herzer. Mater. Sci. Eng. A 133, 1 (1991).

[11] A. Hernando, T. Kulik. Phys. Rev. B 49, 7064 (1994).

[12] А.А. Берзин, А.И. Морозов, А.С. Сигов. ФТТ 58, 1947 (2016).

[13] А.А. Берзин, А.И. Морозов, А.С. Сигов. ФТТ 59, 2420 (2017).

[14] А.И. Морозов, А.С. Сигов. Письма в ЖЭТФ 90, 818 (2009).

Редактор Т.Н. Василевская 